\title{
Dissociation pathways of the p53 DNA binding domain from DNA and critical roles of key residues elucidated by $\mathrm{dPaCS}-\mathrm{MD} / \mathrm{MSM}$
}

\author{
Mohamed Marzouk Sobeh ${ }^{1,2}$ and Akio Kitao ${ }^{1 *}$ \\ ${ }^{1}$ School of Life Science and Technology, Tokyo Institute of Technology, 2-12-1 Ookayama, \\ Meguro-ku, Tokyo 152-8550, Japan \\ ${ }^{2}$ Physics Department, Faculty of Science, Ain Shams University, 11566, Cairo, Egypt \\ *Corresponding author: Akio Kitao, School of Life Science and Technology, Tokyo Institute of \\ Technology, M6-13, 2-12-1 Ookayama, Meguro-ku, Tokyo 152-8550, Japan \\ Tel: +81-3-5734-3373, Fax:+81-3-5734-3372, E-mail: akitao@bio.titech.ac.jp
}




\begin{abstract}
The dissociation process of the DNA binding domain of p53 (p53-DBD) from a DNA duplex that contains the consensus sequence, which is the specific target of p53-DBD, was investigated by a combination of dissociation parallel cascade selection molecular dynamics (dPaCS-MD) and the Markov state model (MSM). Based on an all-atom model including explicit solvent, we first simulated the p53-DBD dissociation processes by 75 trials of $\mathrm{dPaCS}-\mathrm{MD}$, which required an average simulation time of $11.2 \pm 2.2 \mathrm{~ns}$ per trial. By setting the axis of the DNA duplex as the Zaxis and the binding side of p53-DBD on DNA as the + side of the X-axis, we found that dissociations took place along the $+X$ and $-Y$ directions ( $-Y$ directions) in $93 \%$ of the cases, while $7 \%$ of the cases moved along $+\mathrm{X}$ and $+\mathrm{Y}$ directions ( $+\mathrm{Y}$ directions). Toward the $-\mathrm{Y}$ directions, p53-DBD dissociated first from the major groove and then detached from the minor groove, while unbinding from the minor groove occurred first in dissociations along the $+Y$ directions. Analysis of the free energy landscape by MSM showed that loss of the minor groove interaction with p53DBD toward the $+Y$ directions incurred a relatively high energy cost $(1.1 \mathrm{kcal} / \mathrm{mol})$ upon a critical transition, whereas major groove detachment more frequently occurred with lower free energy costs. The standard binding free energy calculated from the free energy landscape was $-10.9 \pm 0.4$ $\mathrm{kcal} / \mathrm{mol}$, which agrees with an experimental value of $-11.1 \mathrm{kcal} / \mathrm{mol}$. These results indicate that the $\mathrm{dPaCS}-\mathrm{MD} / \mathrm{MSM}$ combination can be a powerful tool to investigate dissociation mechanisms of two large molecules. Minor groove binding is mainly stabilized by R248, identified as the most important residue that tightly binds deep inside the minor groove. Analysis of the p53 key residues for DNA binding indicates high correlations with cancer-related mutations, confirming that impairment of the interactions between $\mathrm{p} 53-\mathrm{DBD}$ and DNA can be frequently related to cancer.
\end{abstract}




\section{INTRODUCTION}

p53 was first discovered and considered as an oncogene in 1979, but its main role was identified in the 1990 s to be as a tumor suppressor ${ }^{1,2}$. p53 is a transcriptional factor that regulates cell response to a variety of stresses, including DNA damage, hypoxia, oncogene activation, and other stress signals $\mathrm{s}^{3-5}$. As a transcription factor, p53 binds to DNA promoters in a sequencespecific manner and initiates the transcription of genes involved in cell cycle arrest, apoptosis, and DNA repair 6,7 . Binding of $p 53$ to specific DNA sequences is necessary to activate gene expression ${ }^{5,8}$, indicating the importance of the p53 DNA binding domain (p53-DBD) (residues 94 $292)$ to its function compared to the other functional domains of p53: the transactivation (1-44), tetramerization (325-356), and the C-terminal (357-393) domains ${ }^{9}$. p53-DBD consists of a $\beta$ sandwich scaffold and a DNA-binding domain that includes a loop-sheet-helix (LSH) containing Loop 1 (L1), and two large loops (L2 and L3) stabilized by a zinc atom bridging one His and three Cys residues. The LSH motif and these loops form the p53 DNA-binding surface and contact with the DNA minor and major grooves, respectively, and intermediate regions between them. However, the accumulation of mutations in p53-DBD can cause destabilization and unfolding of the structure, resulting in loss of p53 function as a "guardian of the genome" by inhibiting the ability of p53 to recognize and bind to its target, specific sequence, which has the general form RRRCWWGYYY $(\mathrm{R}=\mathrm{A} / \mathrm{G}, \mathrm{W}=\mathrm{A} / \mathrm{T}, \mathrm{Y}=\mathrm{C} / \mathrm{T})^{9}$ and is conserved in all organisms (consensus sequence). Since p53DBD specifically recognizes the consensus sequence, mutations in p53-DBD can result in oncogenicity ${ }^{10,11}$.

About a half of all human tumors are thought to contain p53 mutations. According to the IARC TP53 Database (https://p53.iarc.fr) ${ }^{12}$, p53 has many mutations (approximately 30,000), of which $\sim 75 \%$ are single missense mutations. In addition, $\sim 95 \%$ of the p53 mutations occur in p53$\mathrm{DBD}^{12-14}$, indicating the potential importance of understanding the roles of p53-DBD key residues in DNA binding. The p53-DBD mutations are classified into two groups. One group affects the 3D-structure of the $\mathrm{p} 53$ native conformation important for its defensive role, and these mutations are termed "structural mutations". The other group affects the direct contact between p53 and DNA, and these mutations are called "contact mutations". Reportedly, 30\% of all p53-DBD mutations occur in six well-known "hotspot" mutation sites. Of these, R248 (L3) and R273 (LSH) are contact mutations and R175 (L2), G245 (L3), R249 (L3), and R282 (LSH) are structural mutations ${ }^{9,15}$. The two contact mutations have the highest mutation rates and are associated with more aggressive malignancies, which indicates the vital role of retaining the binding ability of p53-DBD to DNA to prevent oncogenesis ${ }^{8,16}$. Consequently, elucidating the roles of these residues in stabilizing the p53-DBD/DNA complex structure and the energy of binding of the complex are essential for understanding the sequence recognition mechanisms of the specific DNA consensus sequence by p53-DBD. These mechanisms remain unclear. Thus, more detailed information is required on the essential residues that maintain sequence-specific binding of p53-DBD with DNA. In addition, understanding the association/dissociation processes of p53-DBD to DNA would provide important information on the mechanisms of binding and the key residues that control binding.

Molecular dynamics (MD) simulation is a standard technique to study the structure and function of biological macromolecules, to comprehend the mechanisms underlying complex processes, to analyze experimental findings, and to make predictions ${ }^{17-19}$. MD simulation can provide a comprehensive and quantitative interpretation of protein-protein, protein-DNA, and protein-ligand interactions. In particular, MD simulation allows the calculation of free energy landscapes (FEL), which can quantify interactions between biological molecules ${ }^{20}$. A typical MD simulation with atomistic empirical force fields that includes explicit solvent (all-atom MD) can 
investigate a variety of biological processes that occur within a relatively short timescale, including conformational change ${ }^{21-23}$, ligand binding ${ }^{24}$, and fast folding events of proteins ${ }^{25,26}$. Unfortunately, other vital biological events, such as protein-ligand, protein-protein, and protein-DNA binding/unbinding, which represent most protein folding events, as well as protein aggregation, often occur over much longer timescales and cannot generally be observed by all-atom MD simulations. This is because MD simulation is crucially limited to conformational sampling around specific energy minima that are unable to overcome high energy barriers in a reasonable computational time ${ }^{27,28}$. Consequently, biological processes with longer timescales currently are not within full reach of typical all-atom MD simulations. To address this, a large variety of innovative "enhanced sampling methods" have recently been developed to accelerate the observation of these slow processes and of rare events ${ }^{17,27,28}$. Some of these methods apply artificial bias to enhance sampling, and include free-energy perturbation ${ }^{29}$, umbrella sampling ${ }^{30,31}$, replica exchange umbrella sampling ${ }^{32}$, metadynamics ${ }^{33,34}$, steered $\mathrm{MD}^{35}$, accelerated $\mathrm{MD}^{36,37}$, and adaptive biasing force ${ }^{38}$. Other methods can enhance sampling to explore rare events during simulation without applying any biasing force, such as parallel cascade selection molecular dynamics (PaCS-MD) ${ }^{39,40}$, milestoning ${ }^{41,42}$, weighted ensemble ${ }^{43}$, and forward flux sampling ${ }^{44}$. All these approaches, with or without application of an artificial bias, enhance conformational transitions between different states of the system during most biological processes.

In particular, PaCS-MD is a powerful technique for efficiently estimating the standard binding free energy difference $\left(\Delta \mathrm{G}^{\circ}\right)$ of various biological complexes using the Markov state model (MSM) $)^{40,45-47}$ as a tool for analyzing the PaCS-MD-generated trajectories. PaCS-MD encompasses cycles of multiple independent parallel short all-atom MD simulations accompanied with selection of initial structures for the next cycle based on a certain quantity. Here, we used the inter-center of mass distance between p53-DBD and DNA (Inter-COM distance, $d$ ) as a quantity for ranking the snapshots generated in each cycle. By repeating a series of cycles for the selected top ranked snapshots, dissociation PaCS-MD (dPaCS-MD) generates structures with larger Inter$\mathrm{COM}$ distances than those found in the previous cycle, which significantly enhances the probability of transitions from the bound to unbound states ${ }^{39,48,49}$. Short MD trajectories, a series of molecular configurations, connect the initial bound and final unbound states along the dissociation pathways, and the MD trajectories from many trials of dPaCS-MD can be combined to generate different dissociation pathways, which mutually overlap in conformational space. Using these trajectories, we can construct an MSM which describes the dynamics of a biochemical process as a sequence of transitions between metastable conformational states (microstates) ${ }^{50-52}$. Our group recently established the $\mathrm{APaCS}-\mathrm{MD} / \mathrm{MSM}$ combination to simulate protein-ligand ${ }^{40}$ and protein-protein fracment ${ }^{45,46}$ dissociation, and to accurately calculate $\Delta \mathrm{G}^{\circ}$ in good agreement with experimental values. Therefore, we were motivated to extend the $\mathrm{dPaCS}-\mathrm{MD} / \mathrm{MSM}$ combination for larger biomolecular complexes such as protein/DNA systems. Here, we investigated the dissociation process of the $\mathrm{p} 53-\mathrm{DBD} / \mathrm{DNA}$ complex by dPaCS-MD/MSM.

The goals of this study can be summarized into three main points. 1) Dissociation simulation of the $\mathrm{p} 53-\mathrm{DBD} / \mathrm{DNA}$ complex to investigate the dissociation process. To our knowledge, this is the first report of a successful simulation of this process, at least using enhanced sampling techniques with all-atom models with explicit solvent. 2) Identification of the key residues of the p53-DBD/DNA binding interface. These residues play major roles in the dissociation process and impact the binding free energy of the complex. 3) Generation of preferred dissociation pathways and calculation of binding free energy, which remain computationally challenging. Using $\mathrm{dPaCS}-\mathrm{MD}$, here we investigated the dissociation process of the p53- 
DBD/DNA complex, and the native and non-native contacts released/formed during dissociation. $\mathrm{PaCS}-\mathrm{MD} / \mathrm{MSM}$ overcomes challenges in simulating the dissociation process of large complexes and enables calculation of the binding free energy of p53-DBD to DNA. The key residues, such as R248 and R280, identified by the contact probability during the dissociation process, play important roles for maintaining tight binding of p53-DBD with DNA. We determined the mechanisms of the two main dissociation pathways of the p53-DBD/DNA complex identified from the FEL obtained by MSM. The $\Delta \mathrm{G}^{\circ}$ value deduced from free energy analysis is in good agreement with the experimental value. The utility of $\mathrm{dPaCS}-\mathrm{MD} / \mathrm{MSM}$ for investigating the direction of dissociation and for reproducing the experimentally measured binding free energy can open new possibilities for future study, relating the effects of mutations to the dissociation pathways and to the binding free energy value.

\section{MATERIALS AND METHODS}

\subsection{Interactions between $\mathrm{p53}$-DBD and the DNA consensus sequence in the crystal structure}

In this work, we investigated the fundamental mechanisms of DNA consensus sequence recognition by p53-DBD and thus focused on the interactions of the p53-DBD monomer with DNA. An earlier study revealed that p53-DBD (residues 80-290) binds to the consensus sequence only as four monomers ${ }^{53}$. We searched the p53-DBD/DNA complex structures deposited in the Protein Data Bank (PDB) and selected the one (PDB ID: 1TSR ${ }^{9}$ ) as a suitable structure for our purpose. 1TSR contains a p53-DBD trimer. One monomer is bound extensively with the consensus sequence (Chain B), a second monomer binds to a non-consensus site on the DNA, and the third does not bind to DNA but makes protein-protein contacts, stabilizing the crystal packing. This complex is thus called a monomeric p53-DNA complex. Of the other complex structures in the database (PDB IDs: $2 \mathrm{GEQ}^{54}, 2 \mathrm{ADY}^{55}, 3 \mathrm{EXL}^{56}$, and $3 \mathrm{KMD}^{57}$ ), $\mathrm{p} 53$-DBD binds with DNA as a dimer in 2GEQ and a dimer of dimers in the other complexes. We chose Chain B of 1TSR, p53DBD residues 94-289, as it forms the most native-like monomer interactions with both the major and minor grooves of the DNA ${ }^{9}$. This p53-DBD monomer binds to a DNA duplex containing the consensus sequence and the complementary strand. The sequences are

$5^{\prime}$-TTTCCTAGACTTGCCCAATTA - -
$3^{\prime}$ - - AAGGATCTGAACGGGTTAATA-5'

where bold characters indicate the decamer consensus sequence. It should be noted that notation of the DNA residue numbers in this paper starts from the $5^{\prime}$ - (T1) to $3^{\prime}$-end (A21) of the upper strand, continues to the $5^{\prime}$-end of the second strand (A22), and finally reaches the $3^{\prime}$-end (A42). The previously mentioned six hotspot mutation sites (contact mutations: R248 and R273; structural mutations: R175, G245, R249, and R282) are indicated in Fig. 1A. The interactions on the binding interface between p53-DBD and DNA are of three types: (1) major groove contacts with LSH; (2) minor groove contacts with L3; and (3) phosphate contacts with L3 and LSH (Fig. 1B). L2 does not make significant interactions with the DNA in the crystal structure, but does form several stable interactions after equilibration in the simulation. R280 and R283 of LSH form hydrogen bonds with the DNA major groove and K120 of LSH forms both hydrogen bonds and $\pi$-cation interactions. R248 of L3 forms hydrogen bonds with the minor groove as well as a salt bridge with a phosphate group, consistent with R248 being the most frequently mutated p53 residue in human cancers and is widely assumed to be involved in DNA binding ${ }^{9,58}$. The phosphate groups of the DNA backbone interact with p53-DBD residues such as K120, R273, and R283 (LSH), and S241 (L3), by forming salt bridges, as shown in Fig. 1B. 


\section{Figure 1.}

A) Crystal structure of $\mathrm{p} 53-\mathrm{DBD}$ (Chain B of PDB ID: 1TSR ${ }^{9}$ ) and six hotspot mutation sites indicated by spheres: two contact (magenta) and four structural mutation sites (pink). The regions important for DNA binding (LSH (green) L2 (brown), L3 (light blue)) are shown. B) Chain B of p53-DBD in complex with the DNA duplex in the crystal structure (PDB ID: $1 \mathrm{TSR}^{9}$ ). The region of the DNA duplex containing the consensus sequence and the complementary strand, and other regions, are shown in cyan and blue, respectively. The orientation of p53-DBD is different from that in A, so as to best visualize the interactions between p53-DBD and DNA. Key residues are shown in red (R residues), yellow (K), blue (S), and white (A). Grey and orange spheres represent phosphorus and oxygen atoms of the phosphate groups interacting with these amino acid residues, respectively. VMD was used to create all the structural images shown in this work ${ }^{59}$.
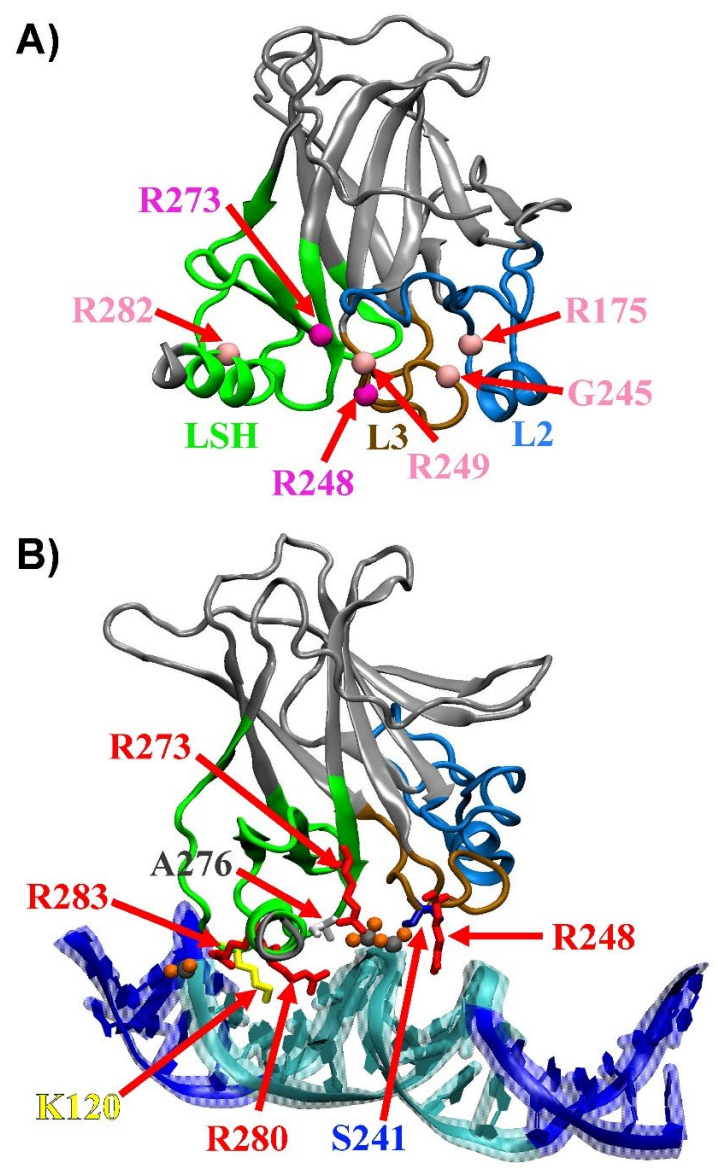

\subsection{Conventional MD simulations}

To generate the initial model of p53-DBD, the N- and C- termini were capped with acetyl (ACE) and N-methlyamide (NME) groups using CHARMM-GUI ${ }^{60}$. Next, we solvated the complex in a $124 \times 109 \times 94 \AA^{3}$ box filled with 4343 TIP3P water molecules ${ }^{61}$. The total charge of the system was then neutralized with $150 \mathrm{mM}$ potassium chloride $(\mathrm{KCl})$ ions to mimic physiological conditions. The axis of the DNA duplex was aligned to the Z-axis so that the 5'-end of the DNA strand with the consensus sequence was oriented toward the $-Z$ direction and $p 53$ $\mathrm{DBD}$ was bound to the $+\mathrm{X}$ direction of the DNA. As the accuracy of the force field model is 
fundamental to successful application of computational methods, the AMBER ff14SB ${ }^{62}$ and OL $15^{63}$ force fields were used for the protein and DNA respectively. As this complex contains a zinc ion, the Zinc AMBER Force Field (ZAFF) ${ }^{64}$ was used to optimize the zinc interface. These preparation steps for the system were performed using the LEaP program of the Amber16 package ${ }^{65,66}$.

To start the simulation, the system was equilibrated in three successive steps. (1) Energy minimization: the energy of the prepared system was minimized by the steepest descent method followed by the conjugate gradient method. (2) NVT equilibration: the temperature of the system was equilibrated at $300 \mathrm{~K}$ for $10 \mathrm{~ns}$ using a Langevin thermostat ${ }^{67}$. This step was conducted under isothermal and isochoric conditions (NVT ensemble). (3) NPT equilibration: the pressure was adjusted to 1 bar using the iso-thermal-isobaric (NPT) ensemble for 10 ns with a Berendsen barostat ${ }^{68}$. We applied positional restraints (force constant: $10 \mathrm{kcal} / \mathrm{mol} \AA^{2}$ ) on the heavy atoms of p53-DBD and DNA during the NVT and NPT equilibration steps. After equilibration, the box dimensions of the system were $118 \times 104 \times 89 \AA^{3}$. During the MD simulation, the SHAKE algorithm ${ }^{69}$ was used to constrain covalent bonds involving hydrogen atoms, and the SETTLE algorithm ${ }^{70}$ was employed to keep the water molecules rigid. The electrostatic interactions were calculated with a real-space cutoff of $10 \AA$ using the particle mesh Ewald (PME) method ${ }^{71}$.

After equilibration, NPT MD simulation of the complex was further conducted for $1 \mu \mathrm{s}$ without any positional restraints to sufficiently sample the conformational space. The MD trajectory was used to inspect the stability of the complex and the distance between p53-DBD and the DNA during the simulation time. Next, clustering was performed using the hierarchical algorithm to choose the most populated cluster as the starting structures for PaCS-MD. GPU implementation of the PMEMD module of the Amber16 package ${ }^{66}$ was used to perform all the MD simulations, with the integration of equation of motion every 2 fs. The analyses were performed using the cpptraj module of the Amber Tools 16 package ${ }^{66}$.

To observe sufficient dissociation, the simulation box of the most populated cluster of the $1 \mu$ s conventional MD simulation was extended to a box of $176 \times 136 \times 136 \AA^{3}$ and the gap was filled with $\sim 95,000$ TIP3 $\mathrm{P}^{61}$ water molecules and $150 \mathrm{mM} \mathrm{KCl}$. The total number of atoms in the system was $\sim 290,000$ atoms, including water molecules and ions. Then, short energy minimization was performed, followed by equilibration at $300 \mathrm{~K}$ and $1 \mathrm{bar}$ for $10 \mathrm{~ns}$. Afterwards, the size of the simulation box was $171 \times 132 \times 132 \AA^{3}$.

\subsection{Dissociation simulation by dPaCS-MD}

PaCS-MD ${ }^{39}$ is an efficient conformational sampling technique that can generate conformational transition pathways using cycles of parallel short MD simulations without applying external force. Each cycle starts by the selection of starting conformations, each of which is used for a distinct replica. For each replica, short-time MD simulation is started with regeneration of the initial velocities with Maxwell-Boltzmann distributions. These cycles are repeated until complete dissociation is achieved. Our group recently succeeded in generating the dissociation pathways of protein/ligand complexes such as tri-N-acetyl-d-glucosamine from hen egg white lysozyme ${ }^{40}$, the transactivation domain of the p53 protein from murine double-minute clone 2 protein $(\mathrm{MDM} 2)^{45}$, and a fragment of the flagellar motor protein FliM from the chemotaxis signaling protein $\mathrm{CheY}^{46}$. In the current work, we extended this method to a larger biomolecular complex, p53-DBD/DNA.

The number of parallel MD simulations (replicas ( $\left.n_{\text {rep }}\right)$ ) typically ranges from 10 to 100 . We selected $n_{\text {rep }}=10$, as this was expected to give sufficient data to build the MSM if trajectories 
from multiple PaCS-MD trials were merged. The snapshots of the trajectories of the current cycle were ranked based on the Inter-COM distance between interface residues of p53-DBD and those of DNA, $d$, and the top 10 snapshots were selected for the next cycle. To decide the optimal choice of defining the interface residues, we conducted trials with different heavy atom-heavy atom distances and found that $5 \AA$ efficiently allowed dissociation. When the Inter-COM distance was defined as the distance between whole molecules, dissociation was also observed but required longer cycles because this distance includes the effect of fluctuations in parts of the protein and DNA other than the interface. In this case, we also observed sliding of p53-DBD along the DNA, but this is beyond the scope of this work and we intend to investigate this observation further in the future.

As the initial structures for PaCS-MD, five snapshots were selected from the last $5 \mathrm{~ns}$ trajectory of the equilibration MD in the large box with 1 ns gap. In other words, we conducted the dissociation simulation from five different initial conformations to obtain more statistics to build the MSM. During dissociation by PaCS-MD, we applied positional restraints (force constant: $1 \mathrm{kcal} / \mathrm{mol} \AA^{2}$ ) for the three base pairs at each end of the DNA strands. This treatment mimics $\mathrm{p} 53-$ DBD binding to a longer DNA duplex, where fluctuations of the ends of the simulated DNA are expected to be less pronounced than in shorter DNA sequences. For each of the top 10 snapshots, a $0.1 \mathrm{~ns}$ MD simulation was performed, and the trajectory was recorded every $200 \mathrm{fs}$, and thus each trajectory contained 500 frames. Finally, we repeated the cycles until $d=70 \AA$ to complete the dissociation. For each snapshot, we conducted 15 trials of PaCS-MD dissociation. Therefore, the total number of trials collected was 75 , which is equal to 5 (restart files) $\times 15$ (the number of PaCS-MD trials for each restart file).

\subsection{Analysis of p53-DBD/DNA interactions}

Since the inter-molecular interactions at the binding interface of p53-DBD and DNA are essential for stabilizing the complex, we compared the interactions of all five different conformations in solution with those in the crystal structure. We also investigated the interactions for the intermediate conformations during the dissociation process, which might give insights into key residues that bind longer with the DNA than other residues. We used Protein Ligand Interaction Profiler (PLIP) to define these interactions and easily identify nonbonded interactions between $\mathrm{p} 53-\mathrm{DBD}$ and $\mathrm{DNA}^{72}$. Also, we checked the top 50 missense mutations, as ranked by their frequencies in diverse human cancers derived from human cancers in the IARC TP53 database $\mathrm{R} 18^{12,58}$, to check whether losing any of these interactions might cause cancer.

\subsection{Free energy analysis by MSM}

The Markov state model (MSM) is widely used in computational biology as a technique to identify stationary states and afford kinetic information on protein dynamics from molecular dynamics simulation data ${ }^{50}$. The MSM can provide important insights into the mechanisms of biological processes because it highlights transitions between microstates ${ }^{73}$, in our case providing insights into dissociation pathways. The dissociation pathways of p53-DBD from the DNA generated by PaCS-MD were analyzed by MSM, as shown previously by our group ${ }^{45}$. Since MD simulations of a new cycle originate from snapshots of the previous cycle, the unbiased trajectories from one trial of PaCS-MD overlap in conformational space along the dissociation pathway. By generating a large number of PaCS-MD pathways, trajectories from different trials also closely overlap, enabling construction of an MSM that covers larger conformational space. Here, for the merged trajectories of 75 trials of PaCS-MD, we built an MSM model based on three-dimensional 
(3D) Inter-COM vector coordinates between p53-DBD and DNA interface residues, hereafter referred to as 3D-MSM. In other words, the COM position of the DNA interface residues was employed as the origin of the $3 \mathrm{D}$ coordinates, and the relative COM position of the p53-DBD interface residues was used as the coordinates for the MSM. The 3D-MSM protocol is established, and experimental values of $\Delta \mathrm{G}^{\circ}$ and kinetic rates are well reproduced ${ }^{45}$. We examined the rootmean-square deviation (RMSD) of p53-DBD and DNA during the dissociation simulations. The RMSD values were mostly in the range of $1-2 \AA$, indicating that the conformational changes of both p53-DBD and DNA were relatively small. This result also supports our assumption that the free energy change of dissociation can be mainly characterized by the position of p53-DBD relative to the DNA.

After choosing 3D COM as the metric, the next step to building the MSM is clustering the MD snapshots into microstates using the appropriate clustering algorithm. Construction of the 3DMSM used the snapshots with Inter-COM distances $d \leq 65 \AA$. Here, we employed k-means ${ }^{74}$ with an initial guess of the cluster center position using k-means $++{ }^{75}$. We tried different numbers of cluster centers until a reasonable result was obtained with 800 cluster centers. To determine the best lag time (or observation interval) for the MSM model, we constructed MSMs with various lag times and investigated the relation between the implied time scales (ITS) versus lag time. ITS of the slowest 100 processes at different lag times is shown in Fig. S1. Based on this analysis, a lag time of 50 ps was identified as the best value to provide Markovian behavior. Next, the transition probability matrix was estimated, followed by calculation of the stationary probabilities of the microstates, as described in the literature ${ }^{73}$. 3D-MDM provides the stationary probability of the microstate $i$ in $3 \mathrm{D}$ space as $p_{i}(X, Y, Z)$, and is converted to the FEL as $-\mathrm{k}_{\mathrm{B}} \mathrm{T} \ln p_{i}(X, Y, Z)$ where $\mathrm{k}_{\mathrm{B}}$ is the Boltzmann constant and $\mathrm{T}$ is the absolute temperature. We used the PyEMMA package ${ }^{76}$ to construct 3D-MSM.

A free energy difference between two states depends only on the starting state and the end state. Therefore, binding free energy is defined as the free energy difference between the bound and unbound states. Although the bound state is a well-defined complex state, the unbound state can be any state in which interactions between p53-DBD and DNA are negligible. Even in the cases where p53-DBD dissociated to very different positions, all dissociated states are equivalent in free energy and all can be considered as unbound states as long as interactions between the protein and DNA can be ignored. The calculation methods for standard binding free energy based on the 1D free energy profile are well established ${ }^{77,78}$. The bound and unbound states are defined from the FEL. Using the probabilities of the bound $\left(\mathrm{P}_{\mathrm{b}}\right)$ and unbound $\left(\mathrm{P}_{\mathrm{u}}\right)$ states, the binding free energy difference from the potential of mean force $(\mathrm{PMF}), \Delta \mathrm{GPMF}$, was calculated by

$$
\Delta \mathrm{G}_{\mathrm{PMF}}=-\mathrm{k}_{\mathrm{B}} \mathrm{T} \ln \frac{\mathrm{P}_{\mathrm{b}}}{\mathrm{P}_{\mathrm{u}}}
$$

To calculate the standard free energy difference of binding, $\Delta \mathrm{G}^{\circ}, \Delta \mathrm{GPMF}$ is corrected by adding the volume correction ${ }^{77}$, which corresponds to the free energy of taking p53-DBD from the standardstate volume $\mathrm{V}^{\mathrm{o}}=1661 \AA^{3}$ (1 M solute concentration) to the sampled unbound volume $\mathrm{V}_{\mathrm{u}}$. The value of $\Delta \mathrm{G}^{\circ}$ is estimated as follows:

$\Delta \mathrm{G}^{\mathrm{o}}=\Delta \mathrm{G}_{\mathrm{PMF}}-\mathrm{k}_{\mathrm{B}} \mathrm{T} \ln \left(\frac{\mathrm{V}_{\mathrm{u}}}{\mathrm{V}^{\mathrm{o}}}\right)$

where the second term is the free energy of the volume correction. The volume of the convex hull defined by the 3D COM coordinates of p53-DBD in the unbound state relative to the DNA was 
used as the value of $V_{u}$ using Qhull ${ }^{79}$. The obtained values of $V_{u}, P_{b}$, and $P_{u}$ were used for the $\Delta G^{\circ}$ calculation.

\section{RESULTS AND DISCUSSION}

\subsection{Structure of the p53-DBD monomer/DNA complex in equilibrium}

We first examined the stability of the p53-DBD monomer/DNA complex during a $1 \mu \mathrm{s}$ MD. The RMSD of the heavy atoms of $\mathrm{p} 53-\mathrm{DBD}$ and DNA from the starting conformation during the $1 \mu \mathrm{s}$ MD was calculated, as shown in Fig. S2A. The RMSD of p53-DBD slightly fluctuated around 2-3 $\AA$, indicating the stability of p53-DBD during the simulation, whereas the RMSD of DNA was larger ( $3-5 \AA$ ). This is consistent with the B-factor values of DNA being roughly 3 -fold that of p53-DBD in 1TSR, indicating larger fluctuations of DNA by 1.7-fold (square root of 3) in amplitude. The larger conformational change of DNA may be related to the removal of two monomers in the crystal form of 1TSR, which contains trimers packed together around the DNA, as well as to the solution environment in the simulation compared to that in the crystal. A $20^{\circ}$ bend in DNA is induced by binding of the $553-\mathrm{DBD}$ dimer ${ }^{54}$. Also, partial disordering of the DNA ends was reported during a structure refinement calculation upon solvation of the crystal structure of a p53 core domain tetramer assembled on full consensus sites without any constraints on the DNA ${ }^{57}$. It should be noted that a significant change in the DNA RMSD was observed around 240-310 ns and is correlated with a sudden change of the Inter-COM distance between the interface residues of p53-DBD and DNA, $d$, from $\sim 13 \AA$ to a stable value of around $8 \AA$ during the remainder of the simulation (Fig. S2B). This indicates tighter binding of p53-DBD with the DNA after the transition. Interestingly, the RMSD of p53-DBD did not particularly change in this time range, showing that p53-DBD binds with the DNA deeper after the transition, changing the DNA structure and interactions between $\mathrm{p} 53-\mathrm{DBD}$ and DNA without significant change in the p53-DBD conformation. The corresponding changes in intermolecular interactions are reported in section 3.2 .

\section{2. $\mathrm{p53}-\mathrm{DBD} / \mathrm{DNA}$ interactions before dissociation}

After the $1 \mu$ s conventional MD simulation, the structure of the most populated cluster was equilibrated in an extended box with sufficient space to accommodate the dissociation of p53DBD from the DNA, as described in section 2.2. Five different conformations around the most stable structure during the MD simulation were then chosen as starting conformations for PaCS$\mathrm{MD}$, as mentioned in section 2.3.

Since multiple starting conformations should be used for efficient sampling ${ }^{23}$, we chose different conformations for the initial structure of $\mathrm{dPaCS}-\mathrm{MD}$ rather than relying only on different initial velocities for one conformation to sample a wider range of $\mathrm{p53-DBD}$ dissociation pathways from DNA. Before starting the dissociation process, we identified the key residues in the binding interface of $\mathrm{p} 53-\mathrm{DBD} / \mathrm{DNA}$ by $\mathrm{PLIP}^{72}$, which revealed differences from the crystal structure.

As shown above, the structure of the p53-DBD/DNA complex was slightly changed compared to the crystal structure. We investigated the interactions between p53-DBD and DNA for the five starting conformations of PaCS-MD by PLIP and compared them to those in the crystal structure, as shown in Table 1 . These changes mainly occurred as the result of the aforementioned tighter binding of p53-DBD with the DNA at around 240-310 ns. R248, R273, R280, and R283 bind to the DNA during MD as well as in the crystal, suggesting their importance for binding. At the major groove, K120 was altered by its neighbor S121 in the MD simulation. This result is consistent with other p53-DBD structures, in which K120 and other residues of the L1 loop in LSH 
do not make a significant contribution to DNA binding ${ }^{54,57}$. At the minor groove, in addition to R248, R249 interacts with the DNA, and this is expected to play an important role in binding because the R249S mutation (PDB code: 2BIO) changes the structure of the L3 loop changed, and DNA binding affinity is drastically reduced ${ }^{80,81}$. Q165 and S166 make contacts with the phosphates but no mutation related to these residues is listed among the 50 most common missense mutations ${ }^{58}$. Below, we discuss the roles of these two residues.

Table 1.

Comparison of p53-DBD residues that make contacts with DNA in at least four out of five starting conformations obtained by MD simulation and in the crystal structure, 1TSR ${ }^{9}$.

\begin{tabular}{|c|c|c|c|}
\hline & \multicolumn{3}{|c|}{ Type of contact } \\
\hline & Major groove & Minor groove & Phosphate \\
\hline Both & R280, R283 & R248 & R248, R273, R283 \\
\hline Only in MD & S121 & R249 & Q165, S166 \\
\hline Only in 1TSR & K120 & - & K120, S241 \\
\hline
\end{tabular}

\section{3. p53-DBD dissociation pathways from DNA}

We successfully generated 75 different dissociation pathways of p53-DBD from DNA by conducting 15 trials of dPaCS-MDs from each of five starting conformations. Figure 2 shows $d$ as a function of the number of PaCS-MD cycles. To assure complete dissociation, each trial was continued until $d$ reached $70 \AA$, which required an average of $112 \pm 22$ cycles (the value after ' \pm ' shows the standard deviation) and 11.2 $\pm 2.2 \mathrm{~ns}$ of dPaCS-MD time. Since each cycle contains 10 parallel MD simulations for $0.1 \mathrm{~ns}$, the accumulated computational cost is $8.4 \mu \mathrm{s}(0.1 \mathrm{~ns} \times 10 \times$ $112 \times 75)$. The variation in the number of cycles is relatively large due to the use of 10 replicas, as we previously showed ${ }^{40}$.

The dissociation of the p53-DBD/DNA complex can be divided into three stages: bound, partially bound, and unbound states. In the bound state ( $d \leq 15 \AA$; below the dotted line in Fig. 2), major key interactions between $\mathrm{p} 53-\mathrm{DBD}$ and DNA were maintained, whereas a part of p53-DBD dissociated from the DNA in the partially bound state $(15 \geq d>35 \AA$, between the dotted and dashed lines in Fig. 2). In the unbound state ( $d>35 \AA$; above the dashed line), p53-DBD completely dissociated, and $d$ increased linearly until it reached the threshold value. 


\section{Figure 2.}

Inter-COM distance between $\mathrm{p} 53-\mathrm{DBD}$ and DNA, $d$, as a function of the number of PaCS-MD cycles for 75 trials. The values of $d$ are plotted only for the replica per cycle whose change in $d$ is the largest among the 10 replicas. Each of the five starting conformations is colored differently, with these colors matching the colors used for the dissociation pathways shown in Fig. 3. The dotted and dashed lines indicate the borders between the bound, partially bound, and unbound states.

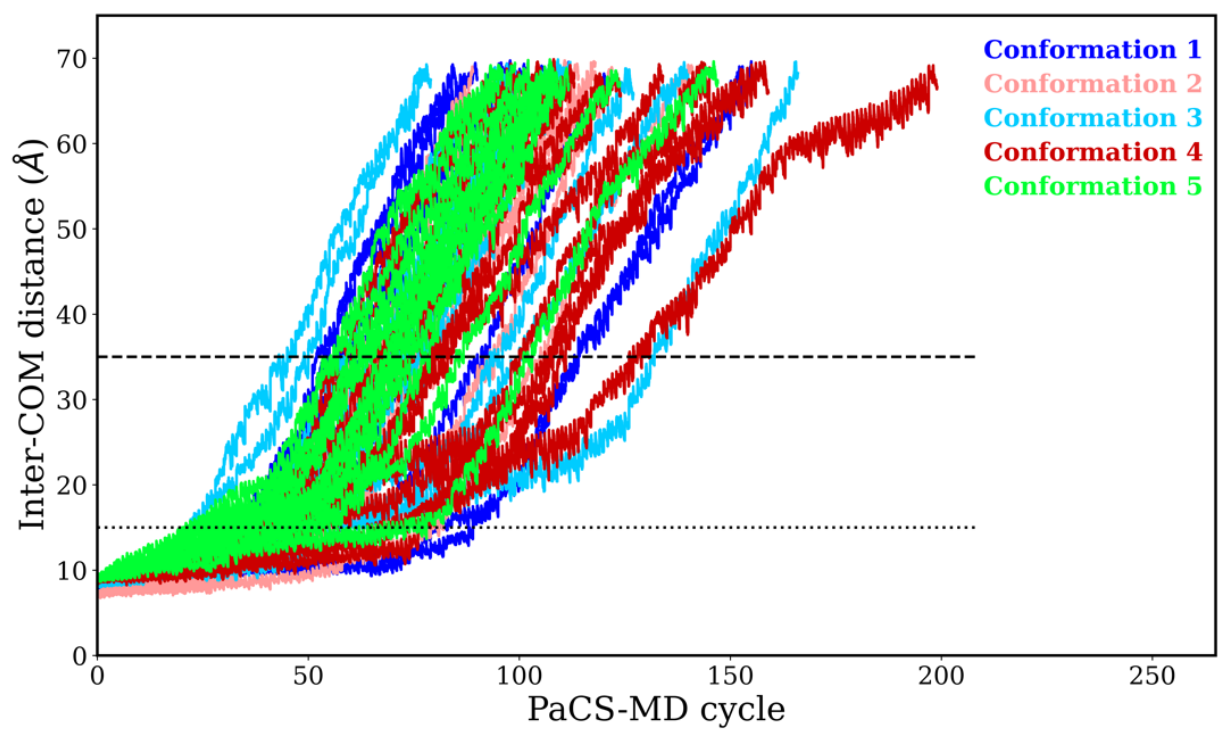

To visualize the 75 dissociation pathways of p53-DBD from the DNA, we used the COM positions of the p53-DBD interface residues relative to those of DNA, as shown in Fig. 3. Inspection of the dissociation pathways indicates that the sampled space formed a cone-like shape around the DNA. Around $93 \%$ of the sampling pathways (70 pathways) dissociated along the $+X$ and $-Y$ directions (namely, the $-Y$ directions), while the other pathways moved along $+X$ and $+Y$ directions, which only occupy $7 \%$ (5 pathways), hereafter called the $+Y$ directions. Therefore, the $\mathrm{X}$-axis is considered to correspond to the main reaction coordinate for dissociation, while the other two coordinates (corresponding to the $\mathrm{Y}$ - and $\mathrm{Z}$-axes) are considered as secondary coordinates.

To investigate the main differences between the $-Y$ and $+Y$ directions, we inspected the trajectories of both directions in detail. The first trajectory represents a typical dissociation pathway along the $-\mathrm{Y}$ directions (see Movie S1), while the second trajectory represents that along the $+Y$ directions (Movie S2). We noticed that toward the $-Y$ directions, $p 53-D B D$ dissociated from the DNA major groove and subsequently dissociated from the minor groove. In contrast, along the $+Y$ directions, p53-DBD first dissociated from the minor groove and later dissociated from the major groove. 


\section{Figure 3.}

Dissociation pathways of 75 PaCS-MD trials of p53-DBD (pink cartoon model) from DNA (blue) represented by the COM positions of $\mathrm{p} 53$-DBD relative to the DNA in the trajectories. The coloring of the pathways is identical to that in Fig. 2. A) Front view and B) view rotated by $90^{\circ}$ around the $\mathrm{X}$-axis (in the YZ-plane).

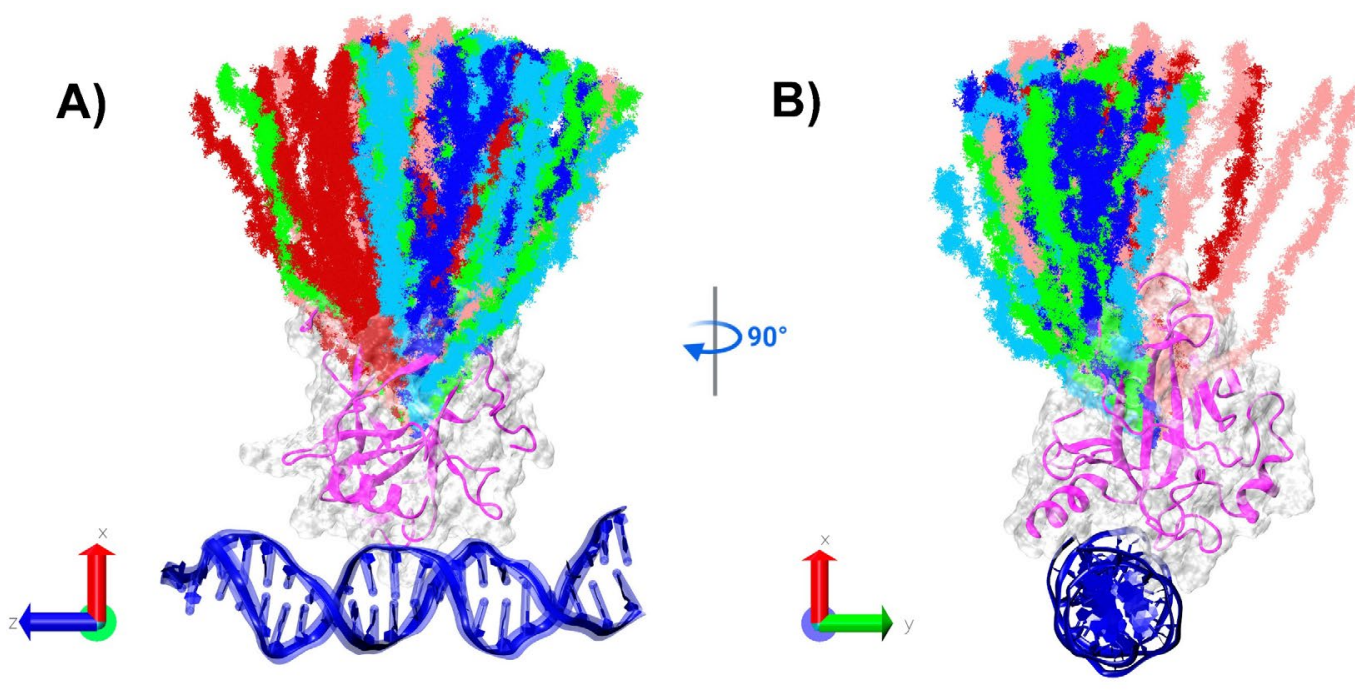

\subsection{Key interactions during the dissociation process and their relation to cancer mutations}

To obtain insights into the essential interactions between p53-DBD and DNA during dissociation, we calculated the contact probabilities for 41 contact pairs of p53-DBD and DNA residues as a function of the Inter-COM distance $d$. These contact pairs were selected from the pairs of p53-DBD and DNA residues whose inter-atom distances were within $3.5 \AA$ for at least one pair of atoms in at least one snapshot among the equilibrated trajectories just before the $1^{\text {st }}$ cycle of the PaCS-MD trials. To calculate the contact probabilities, we generated a reactive trajectory for each trial of PaCS-MD, comprising a series of molecular configurations connecting the initial bound and final unbound states along the dissociation pathway, and calculated the probabilities from all the 75 trials with a bin size of $1 \AA$ as a function of $d$. The result for all 41 pairs is shown in Fig. S3 for the range of bound and partially bound states $(d \leq 35 \AA)$. Of these pairs, we selected 19 native contact pairs that include the pairs that started with high probabilities ( $>80 \%$ ) and which occupy $46 \%$ of all the pairs (Fig. 4A) and two transient contact pairs whose probabilities were low in the bound state $(<20 \%)$ but increased to around $30 \sim 40 \%$ before and after the border of the bound and unbound states, as shown in Fig. 4B. We noticed that $63 \%$ of the native contact pairs (12 pairs) contain an Arg residue of p53-DBD. Arginine is an amino acid residue essential for binding that has a positively charged guanidinium group at the end of a polar region, making it suitable for binding a phosphate anion, a main constituent of DNA strands. This also explains why five of the top six hotspot mutation sites are Arg residues, all of which are situated in the p53-DBD/DNA interface bound to the DNA consensus sequence (Fig. 1A). 


\section{Figure 4.}

Contact probabilities as a function of the Inter-COM distance between p53-DBD and DNA, $d$. A) 19 native contact pairs whose initial probabilities were greater than $80 \%$. B) Two transient pairs whose initial probabilities were smaller than $20 \%$ but showed a significant increase during the dissociation process.

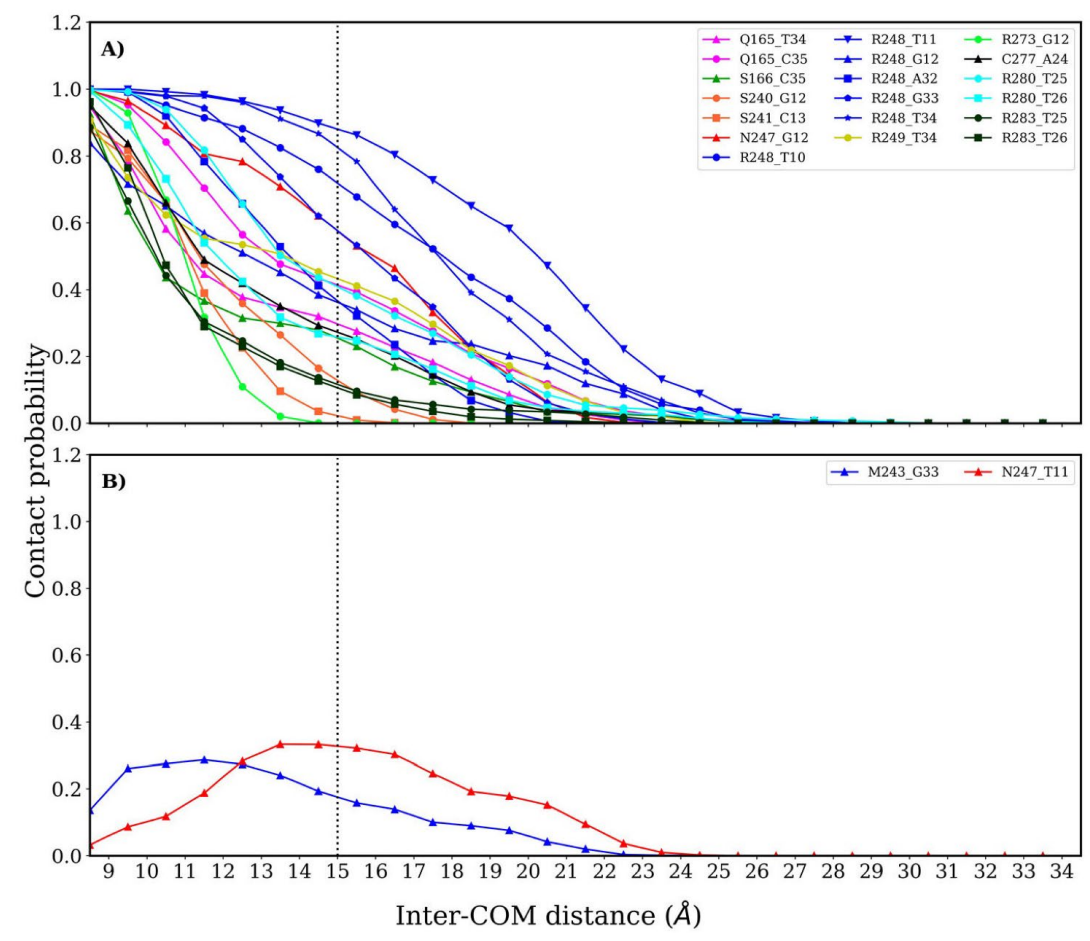

R248 and R273 are the two most highly mutated sites in the p53 protein (6.79 and $6.55 \%$ respectively) ${ }^{12}$ and both are considered as contact residues, as shown in Fig. 1. We found that R248 maintained binding with the DNA base pairs for a much longer time before complete dissociation. R248 was identified as the last residue to dissociate from DNA in $75 \%$ of the trials (56 cases out of 75). On the other hand, R273 completely dissociated at $d=14 \AA$ within the bound state. This indicates the major role that R248 plays to maintain the tight binding of p53-DBD to the DNA until the final step of the dissociation process. As mentioned in section 2.1, R248 is situated in L3 and is bound to a deep part of the DNA minor groove, and is stabilized by a variety of non-bonded interactions with the DNA. This residue is situated in this position, contributing to the stabilization of $\mathrm{L}^{82}$. A closer look revealed that R248 binds to the DNA minor groove by seven different contacts with three nucleotide residues (T10, T11, and G12) from one strand and with four (A31, A32, G33, and T34) from the other strand (Fig. S3). Six of these contacts are considered as the native contacts. It should be noted that T10-G12 are in the middle of the consensus sequence (A7C16), indicating the central role of R248 in sequence recognition. Three of the contacts (R248T10, R248-G12, and R248-T34) are maintained with probabilities around 30\% until $d=20 \AA$, while the other three interactions (R248-T11, R248-A32, and R248-G33 tend to be lost at shorter $d$. These findings could explain why the R248Q mutation, which is both a contact and a structural mutation $^{83}$, causes a decrease in binding affinity to DNA. The result of MD simulation suggested that the p53 binding affinity to DNA is reduced in the R248Q mutant but that the complex is_not dramatically destabilized $^{84,85}$. In contrast, R273 binds with the DNA major groove ${ }^{57,86}$. R273 only 
has two nonbonded interactions with DNA (R273-T11 and R273-G12) and only one of them showed a probability greater than $80 \%$ and quickly dissociated. This could explain why mutations of R273 (R273H and R273C) do not contribute as much to binding affinity as the mutation of R248 (R248W), but these two mutations together are directly associated with impaired DNA binding, probably due to loss of the arginine guanidinium group ${ }^{87}$.

Although the binding of p53-DBD to the minor groove mainly relies on R248, the neighboring R249 was also identified to bind with DNA in all the five starting conformations. In the 1TSR crystal structure, R249, which is situated in L3, interacts with other parts of the protein, such as L2, L3, and strands of the $\beta$ sandwich, playing a role in stabilizing the p53-DBD structure? In our simulations, due to its neighboring position to R248 in the minor grove, R249 also interacts with T34 of DNA, maintaining 40\% of the binding probability at the beginning of the partially bound state and also playing roles in maintaining binding to the DNA. R249S mutations are extremely common in liver cancer in some developing countries ${ }^{16}$. A change in the size of the side chain and absence of the positively charged guanidinium group in R249S were reported to be the main reasons for the disruption of the R249 hydrogen bond network, ultimately leading to the loss of DNA binding ${ }^{88}$.

R280 and R283 contribute to the binding of p53-DBD with the DNA major groove. R280 forms two hydrogen bonds with the bases of T25 and T26, maintaining contact probabilities around $20 \sim 40 \%$ at $d=17 \AA$. This residue was identified as the last residue to dissociate from DNA in $20 \%$ of trials (15 cases out of 75). This percentage shows that this is the second most frequent residue to be the last residue to dissociate from DNA (the most frequent residue is R248). R283 bound with T25 and T26 dissociated faster (probabilities of $<10 \%$ at the same distance). In some crystal structures, R280 also binds to bases of the major groove and the backbone of DNA and makes invariant contacts with the conserved guanine base ${ }^{57,86}$. As a result, R280 is also classified as a contact mutation site, the same as mutations of R248 and R273. Although R280 is absent from the listed of the hotspot mutations, prior findings showed that R280 is important in direct DNA recognition and that mutation of this residue (R280K) impairs DNA transcription, resulting in various forms of cancer ${ }^{89,90}$. We examined the list of the top 50 missense p53 mutations ranked by their frequencies in diverse human cancers ${ }^{12,58}$ and found that two R280 mutations are below the top 25 and the total low percentage is only around $0.8 \%$. In contrast, R248Q and R248W are ranked in the top five, with a frequency of around $7.9 \%$.

The native contacts were gradually lost during the dissociation process, but two transient interactions were formed, as shown in Fig. 4B. The contact probabilities of M243 and N247 were maximum at $d=11.5 \AA$ and $13.5 \AA$, respectively. N247 maintained binding with G12 of the DNA for a relatively long period of time until it completely lost the contact at $d=25 \AA$ in the middle of the partially bound state. Although the N247 interaction was only detected in one of the five starting conformations (conformation 5), it can form a transient interaction with G33 at around $d$ $=13-16 \AA$ with a probability $>30 \%$. Transient interactions may suppress an abrupt energy change during the dissociation process by forming interactions to support binding.

C277 maintained binding with a $20 \%$ probability until $d=17 \AA$. This residue is a prime binding target for some anti-cancer compounds that attempt to reactivate mutant $\mathrm{p} 53^{91}$. In the $3 \mathrm{KMD}$ crystal structure, C277 contacts with bases of the major groove and the backbone of DNA by different non-bonded interactions ${ }^{57}$. The contacts of $\mathrm{C} 277$ to the major groove vary depending on the DNA sequence ${ }^{86}$. 


\subsection{Free energy landscape (FEL) of dissociation and two dissociation directions}

We obtained the FEL of p53-DBD dissociation from DNA, as described in section 2.5, and showed the projections onto the XY-, XZ-, and YZ-planes, as shown in Figs. 5A, B, and C, respectively. The global minimum of the FEL agrees with the bound state. The bound state is a very deep free energy minimum (red color in FEL) and no other clear minimum is found along the dissociation pathways. As shown in Fig. 3 and Movies S1 and S2, dissociation mainly (93\%) occurred along the $-\mathrm{Y}$ directions on the XY-plane and other dissociations (7\%) occurred along the $+Y$ directions. Therefore, we chiefly focused on the FEL of the XY-plane, shown as Fig. 5A. Although the $-Y$ directions are the major dissociation pathways, free energy changes along these directions are steeper compared to those along the $+\mathrm{Y}$ directions in Fig. 5A. This tendency is also seen as a left-right asymmetry in the FEL of the YZ-plane (Fig. 5C). Compared to Fig. 5A, Fig. $5 \mathrm{~B}$ shows better vertical symmetry around $\mathrm{Z}=\sim 0$ in the FEL of the XZ-plane, indicating equivalence of dissociations along a variety of $Z$ directions.

\section{Figure 5.}

Free energy landscape of p53-DBD dissociation from DNA mapped onto the A) XY-, B) XZ-, and C) YZ-planes. D) The potential of mean force $\mathrm{F}$ as a function of $\mathrm{X}$ obtained by averaging microstate probabilities only in the $-\mathrm{Y}$ area. The obtained values of $\Delta \mathrm{G}_{\mathrm{PMF}}$ and $\Delta \mathrm{G}^{\circ}$ are also shown.
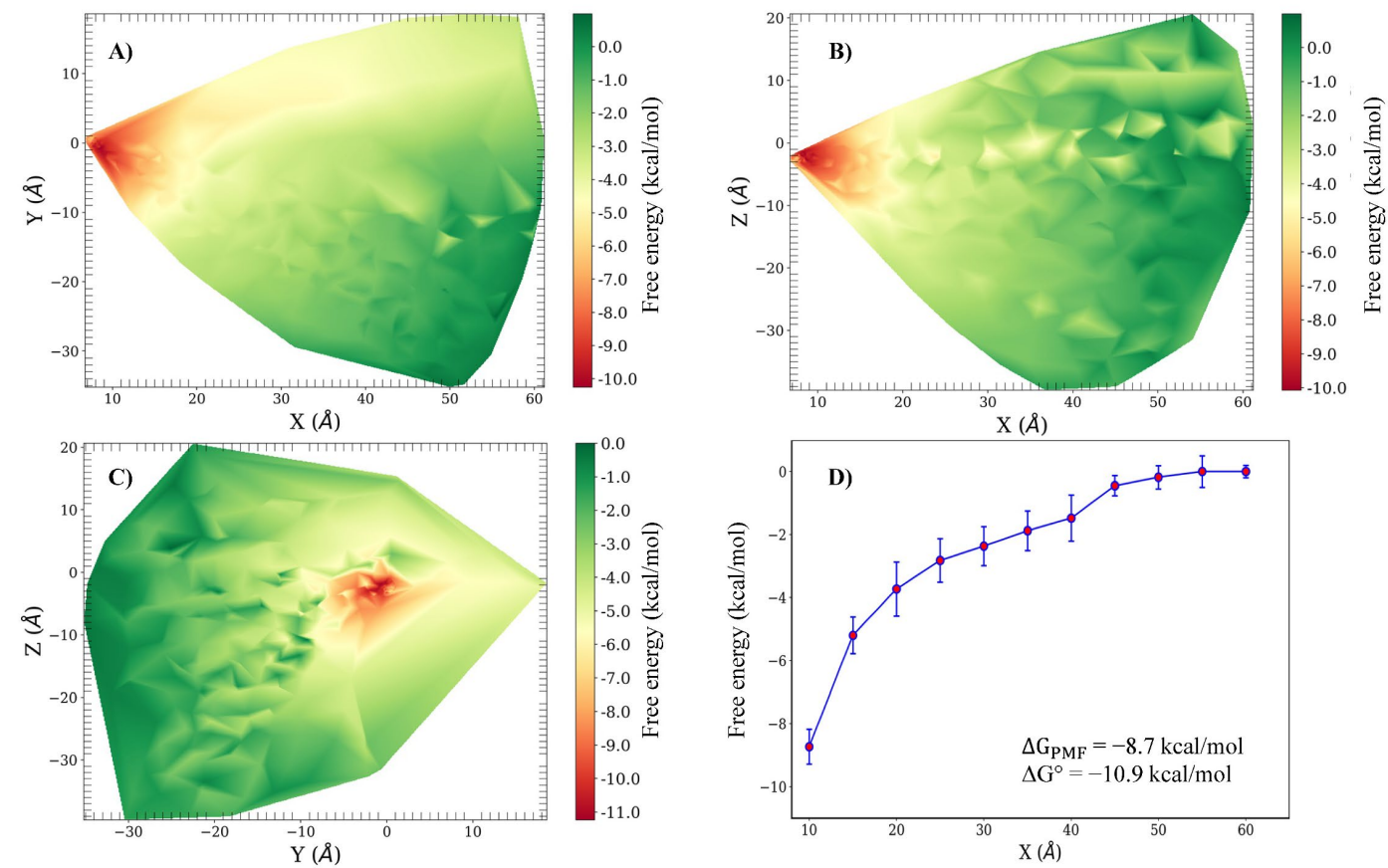

To further investigate the reason for this asymmetry we examined the transition probabilities between microstates around a branch point of the two dissociation directions and the free energy values of microstates, as shown in Fig. 6A and B. The points indicated by $\mathrm{C}$ in these panels show the microstate that can reach to the $+\mathrm{Y}$ directions upon transition to point $\mathrm{D}$, and $\mathrm{D}$ indicates the microstate just after the critical transition toward the $+\mathrm{Y}$ directions. Figures $6 \mathrm{C}$ and D show representative snapshots of these critical microstates. In Fig. 6A, the thickness of the line 
between microstates shows the magnitude of the probability. The transition probability from $\mathrm{C}$ to $\mathrm{D}$ is significantly less than those going to the $-\mathrm{Y}$ directions. This is consistent with a larger free energy gap from $\mathrm{C}$ to $\mathrm{D}(1.1 \mathrm{kcal} / \mathrm{mol})$ toward the $+\mathrm{Y}$ directions compared to those toward the $-\mathrm{Y}$ directions $(\leq 0.5 \mathrm{kcal} / \mathrm{mol})$. These results indicate that, although the $+\mathrm{Y}$ directions show overall shallower free energy changes after the transition to the partially bound state, most dissociations occur along the $-Y$ directions at the branch point where the dissociation along the $-Y$ directions is energetically preferable. As is clear from comparison of Figs. 6C and D, the minor groove contacts were lost upon the transition from $\mathrm{C}$ to $\mathrm{D}$, indicating greater importance of $\mathrm{p} 53-\mathrm{DBD}$ binding to the minor groove. This also indicates larger free energy contributions of minor groove binding compared to the contributions of major groove. As mentioned in section 3.4, R248 plays major roles in minor groove binding by interacting with seven nucleotide residues, including the middle of the consensus sequence, and by bridging the two DNA strands, and R248 was the last residue to detach from the DNA in $75 \%$ of the PaCS-MD trials. Also, R248Q and R248W are ranked in the top five of the top 50 missense p53 mutations, with a frequency of around $7.9 \%$. We conclude that R248 is essential in recognizing the consensus sequence and in stabilizing p53-DBD binding with DNA.

\section{Figure 6.}

A) Transition probabilities between microstates along the dissociation directions visualized by the thickness of the lines in a close-up view of the FEL up to $X \leq 25 \AA$. When the probabilities are lower than 0.001 , no lines are shown. B) Free energy values are shown for the corresponding microstates. C) A representative snapshot of the microstate before the critical transition toward dissociation to the $+Y$ directions and D) a snapshot just after the critical transition. The positions of $\mathrm{C}$ and $\mathrm{D}$ are indicated in panels $\mathrm{A}$ and $\mathrm{B}$. p53-DBD residues contacting DNA are shown in red.
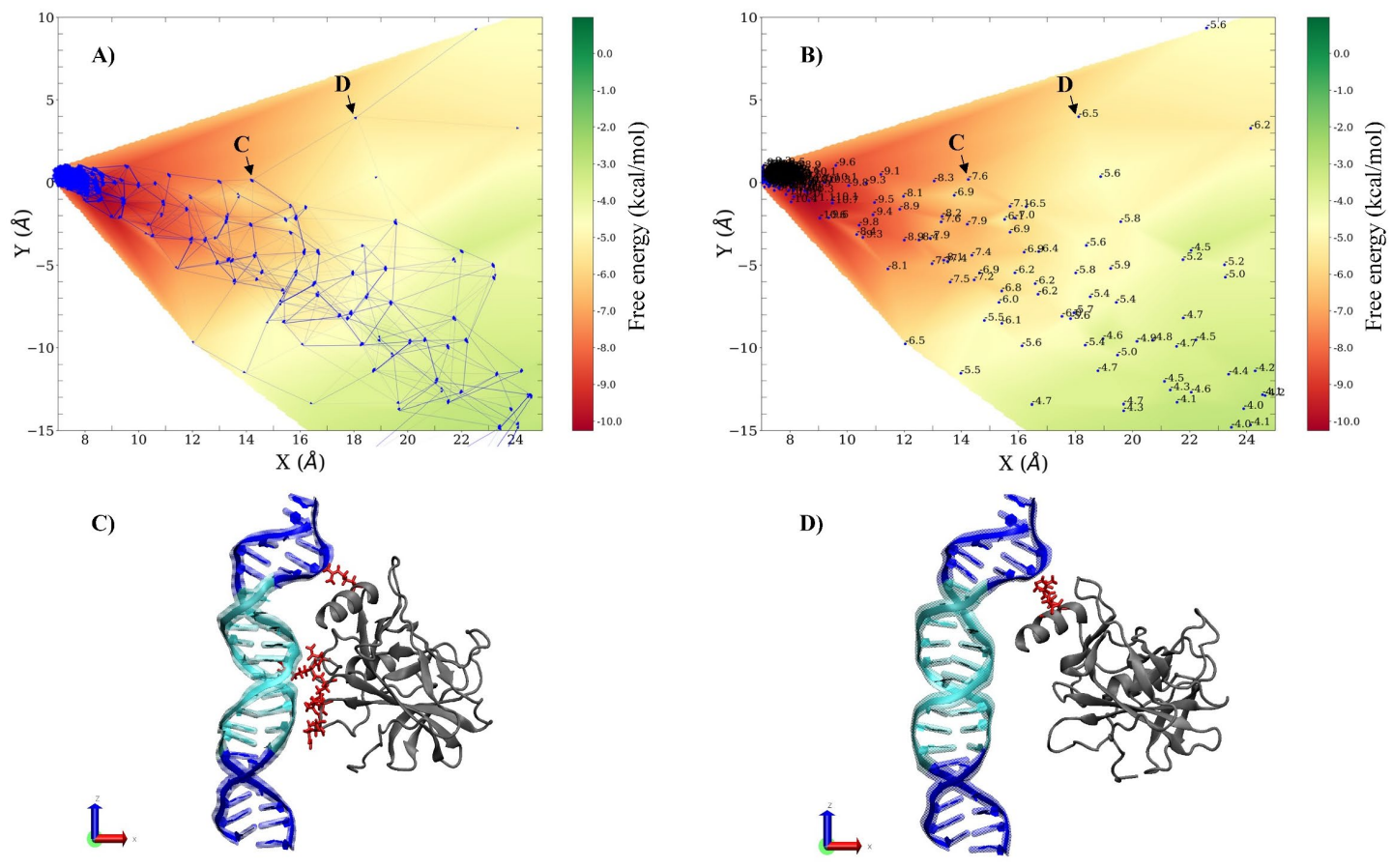
To examine the validity of the calculated FEL, we estimated the standard binding free energy $\Delta \mathrm{G}^{\circ}$ and compared it to the experimental value. As already shown previously in Eq. (2), $\Delta \mathrm{G}^{\circ}$ is obtained from the probabilities of the bound and unbound states with volume correction. The unbound state can be any state in which the FEL reaches a plateau, as mentioned in section 2.5. As shown in Fig. 5A, the FEL converged to flat values along the $-Y$ directions. To illustrate this convergence, the PMF as a function of $\mathrm{X}$ obtained by averaging microstate probabilities only in the $-\mathrm{Y}$ area is shown in Fig. 5D. The distribution of these microstates in the COM space is shown in Fig. S4. During the dissociation process, the PMF increased at a higher rate in the bound state $(d<15 \AA)$, and continued increasing with a lower rate in the partially bound state $(15 \leq d<$ $35 \AA$ ) and became flat in the unbound state, especially at around 45-60 $\AA$. Regardless of the direction of dissociation, the PMF converges to a certain value when two molecules are separated sufficiently and the interactions between these molecules are negligible, as shown previously ${ }^{40,45}$. Thus, the unbound state was defined as the flat region $(\mathrm{d} \geq 45 \AA)$. After calculating the unbound volume of the trajectories, we obtained $\Delta \mathrm{G}^{\circ}=-10.9 \pm 0.4 \mathrm{kcal} / \mathrm{mol}\left(\Delta \mathrm{G}_{\mathrm{PMF}}=-8.7 \pm 0.4\right.$ and the correction value $=-2.2 \mathrm{kcal} / \mathrm{mol}$ ). This value is very close to the binding free energy of $\mathrm{p} 53-\mathrm{DBD}$ (residues 94-312) with the consensus DNA sequence of $-11.1 \mathrm{kcal} / \mathrm{mol}$ measured by isothermal titration calorimetry (ITC) $)^{92}$, suggesting that the calculated FEL is reasonable.

\section{CONCLUSION}

In this work, we observed the dissociation processes of p53-DBD from DNA that contains the consensus sequence in the middle of the sequence by dissociation PaCS-MD (dPaCS-MD) simulations with an all-atom model including explicit solvent. Seventy-five trials of dPaCS-MD were conducted with an average simulation time of $11.2 \pm 2.2 \mathrm{~ns}$. During the dissociation process, $93 \%$ of the trials dissociated along the $+X$ and $-Y$ directions ( $-Y$ directions), while $7 \%$ moved along the $+\mathrm{X}$ and $+\mathrm{Y}$ directions ( $+\mathrm{Y}$ directions). Along the $-\mathrm{Y}$ directions, $\mathrm{p}$ 53-DBD dissociated from the major groove first and then detached from the minor groove, while unbinding from the minor groove occurred first along the $+Y$ directions, followed by dissociation from the major groove. Since the loss of minor groove interaction with p53-DBD has a relatively high free energy $\operatorname{cost}(1.1 \mathrm{kcal} / \mathrm{mol})$ upon the critical transition toward the $+Y$ direction, major groove detachment occurs more frequently with a lower free energy cost $(<0.5 \mathrm{kcal} / \mathrm{mol})$ as the initial step of dissociation. The standard binding free energy calculated from the free energy landscape was $\Delta \mathrm{G}^{\circ}$ $=-10.9 \pm 0.4 \mathrm{kcal} / \mathrm{mol}$, which agrees with the value obtained by ITC, $-11.1 \mathrm{kcal} / \mathrm{mol}$. These results indicate that the $\mathrm{dPaCS}-\mathrm{MD} / \mathrm{MSM}$ approach can be a powerful tool to investigate the dissociation mechanisms of two large molecules, such as a protein and a DNA molecule.

The minor groove binding is stabilized mainly by R248 and R249 and sometimes also by N247. Among them, R248 is the most important residue that tightly packs deep inside the minor groove. This residue contacts with 7 nucleotide residues including the middle of the consensus sequence, bridging the two DNA strands that form the minor groove. Also, R248 was the last residue detached from the DNA in $75 \%$ of the dPaCS-MD trials. These results explain why R248 mutations significantly affect the binding of p53 to DNA, interfere p53 functions, and finally lead to human cancer. R248 is one of the most frequently mutated residues in the top 50 list of p53 missense mutations. Two major mutations, R248Q and R248W, loose the charged side chain that interact with 7 nucleotide residues. Previously, atomistic MD simulations of p53-DBD/DNA also showed that a few crucial residues, specifically R248, S241, and N239, contribute to binding energy by using the molecular mechanics Poisson-Boltzmann surface area (MM-PBSA) ${ }^{85}$. The binding of p53-DBD to the major groove is stabilized by R280 and R283. R280 was the last residue 
dissociated from the DNA in $20 \%$ of trials while R283 tended to dissociate faster. R280 mutations are found in the top 50 list but situated below top 25 and the total low percentage is only around $0.8 \%$. These results show that the $\mathrm{p} 53$ key residues for the DNA binding are highly related to the cancer-related mutations, which confirms that impairments of interactions between p53-DBD and DNA can be frequently related to cancer.

In summary, the promising combination of $\mathrm{dPaCS}-\mathrm{MD} / \mathrm{MSM}$ can be used not only to investigate different pathways during dissociations of two large molecules but also to identify key residues for major dissociation pathways and to quantitatively calculate the binding free energy of the complex, which should also be useful in elucidating the effects of mutations. The presence of allosteric roles and inactivating effects of the p53-DBD mutations located distant from the DNA binding surface that were recently revealed ${ }^{93-95}$ may also be investigated by $\mathrm{dPaCS}-\mathrm{MD} / \mathrm{MSM}$ to quantitatively analyze mutational effects on binding free energy and binding mechanisms in the future. We conclude that this combination sheds light on underlying mechanisms, which are highly necessitated for developing small molecules as anti-tumor drugs that can reactivate functions of p53 mutants.

\section{Author Contributions}

M.M.S. performed the computations. M.M.S. and A.K. contributed to the design of the work, conducted the overall analyses, and wrote the manuscript.

\section{Notes}

The authors declare no competing financial interest.

\section{ACKNOWLEDGEMENTS}

This research was supported by MEXT/JSPS KAKENHI Nos. JP19H03191, JP20H05439, and JP21H05510 to A.K., and by MEXT as a "Program for Promoting Researches on the Supercomputer Fugaku" (Application of Molecular Dynamics Simulation to Precision Medicine Using Big Data Integration System for Drug Discovery, JPMXP1020200201 and Biomolecular Dynamics in a Living Cell, JPMXP1020200101) to A.K. M.M.S. was supported in part by a full $\mathrm{Ph}$.D. scholarship from the Egyptian Ministry of Higher Education, Cultural Affairs and Missions sector according to the Egypt-Japan Education Partnership (EJEP) program. This work used computational resources of the supercomputer TSUBAME provided by Tokyo Institute of Technology, FUGAKU through the HPCI System Research Project (Project IDs: hp210029, hp210172, and hp210177), Research Center for Computational Science, The National Institute of Natural Science, and The Institute for Solid State Physics, The University of Tokyo.

\section{REFERENCES}

(1) Linzer, D. I. H.; Levine, a. J. Characterization of a 54K Dalton Cellular SV40 Tumor Antigen Present in SV40-Transformed Cells and Uninfected Embryonal Carcinoma Cells. Cell 1979, 17 (1), 43-52. https://doi.org/10.1016/0092-8674(79)90293-9.

(2) Oren, M. P53: Not Just a Tumor Suppressor. J. Mol. Cell Biol. 2019, 11 (7), 539-543. https://oi.org/10.1093/jmcb/mjz070.

(3) Bieging, K. T. Unravelling Mechanisms of P53-mediated Tumour Suppression. Nat. Rev. Cancer 2014, 14 (5), 359-370. https://doi.org/10.1038/nrc3711.Unravelling.

(4) Bowen, M. E.; Attardi, L. D. The Role of P53 in Developmental Syndromes. J. Mol. Cell Biol. 2019, 11 (3), 200-211. https://doi.org/10.1093/jmcb/mjy087. 
(5) Goldstein, I.; Marcel, V.; Olivier, M.; Oren, M.; Rotter, V.; Hainaut, P. Understanding Wild-Type and Mutant P53 Activities in Human Cancer: New Landmarks on the Way to Targeted Therapies. Cancer Gene Ther. 2011, 18 (1), 2-11. https://doi.org/10.1038/cgt.2010.63.

(6) Vyas, P.; Beno, I.; Xi, Z.; Stein, Y.; Golovenko, D.; Kessler, N.; Rotter, V.; Shakked, Z.; Haran, T. E. Diverse P53/DNA Binding Modes Expand the Repertoire of P53 Response Elements. Proc. Natl. Acad. Sci. U. S. A. 2017, 114 (40), 10624-10629. https://doi.org/10.1073/pnas.1618005114.

(7) Bykov, V. J. N.; Eriksson, S. E.; Bianchi, J.; Wiman, K. G. Targeting Mutant P53 for Efficient Cancer Therapy. Nat. Rev. Cancer 2018, 18 (2), 89-102. https://doi.org/10.1038/nrc.2017.109.

(8) Wu, W.-S.; Chang, J.-W.; Liaw, H.-J.; Chu, Y.-H.; Jiang, Y.-X. P53 Binding Loci Database (P53BLD): A Repository for the Genome-Wide Binding Loci of Human TP53. Clin. Microbiol. Res. 2019, 1-10. https://doi.org/10.31487/j.cmr.2018.01.01.

(9) Cho, Y.; Gorina, S.; Jeffrey, P. D.; Pavletich, N. P. Crystal Structure of a P53 Tumor Suppressor-DNA Complex: Understanding Tumorigenic Mutations. Science (80-. ). 1994, 265 (5170), 346-355. https://doi.org/10.1126/science.8023157.

(10) Lane, D. P. P53, Guardian of the Genome. Nature 1992, 358, 15--16. https://doi.org/10.1038/358015a0.

(11) Dittmer, D.; Pati, S.; Zambetti, G.; Chu, S.; Teresky, A. K.; Moore, M.; Finlay, C.; Levine, A. Gain of Function Mutations in P53. Nat. Genet. 1993, 4 (1), 42--46. https://doi.org/10.1038/ng0593-42.

(12) Bouaoun, L.; Sonkin, D.; Ardin, M.; Hollstein, M.; Byrnes, G.; Zavadil, J.; Olivier, M. TP53 Variations in Human Cancers: New Lessons from the IARC TP53 Database and Genomics Data. Hum. Mutat. 2016, 37 (9), 865-876. https://doi.org/10.1002/humu.23035.

(13) Martin, A. C. R.; Facchiano, A. M.; Cuff, A. L.; Hernandez-Boussard, T.; Olivier, M.; Hainaut, P.; Thornton, J. M. Integrating Mutation Data and Structural Analysis of the TP53 Tumor-Suppressor Protein. Hum. Mutat. 2002, 19 (2), 149-164. https://doi.org/10.1002/humu.10032.

(14) Petitjean, A.; Achatz, M. I. W.; Borresen-Dale, A. L.; Hainaut, P.; Olivier, M. TP53 Mutations in Human Cancers: Functional Selection and Impact on Cancer Prognosis and Outcomes. Oncogene 2007, 26 (15), 2157-2165. https://doi.org/10.1038/sj.onc.1210302. Lu, Q.; Tan, Y. H.; Luo, R. Molecular Dynamics Simulations of P53 DNA-Binding Domain. J. Phys. Chem. B 2007, 111 (39), 11538-11545. https://doi.org/10.1021/jp0742261.

(16) Brosh, R.; Rotter, V. When Mutants Gain New Powers: News from the Mutant P53 Field. Nat. Rev. Cancer 2009, 9 (10), 701-713. https://doi.org/10.1038/nrc2693.

(17) De Vivo, M.; Masetti, M.; Bottegoni, G.; Cavalli, A. Role of Molecular Dynamics and Related Methods in Drug Discovery. J. Med. Chem. 2016, 59 (9), 4035-4061. https://doi.org/10.1021/acs.jmedchem.5b01684.

(18) Karplus, M.; McCammon, J. A. Molecular Dynamics Simulations of Biomolecules. Nat. Struct. Biol. 2002, 9 (9), 646-652. https://doi.org/10.1038/nsb0902-646.

(19) Arnittali, M.; Rissanou, A. N.; Harmandaris, V. Structure of Biomolecules Through Molecular Dynamics Simulations. Procedia Comput. Sci. 2019, 156, 69-78. https://doi.org/10.1016/j.procs.2019.08.181.

(20) Hedger, G.; Shorthouse, D.; Koldsø, H.; Sansom, M. S. P. Free Energy Landscape of 
Lipid Interactions with Regulatory Binding Sites on the Transmembrane Domain of the EGF Receptor. J. Phys. Chem. B 2016, 120 (33), 8154-8163.

https://doi.org/10.1021/acs.jpcb.6b01387.

(21) Dror, R. O.; Arlow, D. H.; Borhani, D. W.; Jensen, M.; Piana, S.; Shaw, D. E. Identification of Two Distinct Inactive Conformations of the $\beta$ 2-Adrenergic Receptor Reconciles Structural and Biochemical Observations. Proc. Natl. Acad. Sci. U. S. A. 2009, 106 (12), 4689-4694. https://doi.org/10.1073/pnas.0811065106.

(22) Dror, R. O.; Pan, A. C.; Arlow, D. H.; Borhani, D. W.; Maragakis, P.; Shan, Y.; Xu, H.; Shaw, D. E. Pathway and Mechanism of Drug Binding to G-Protein-Coupled Receptors. Proc. Natl. Acad. Sci. U. S. A. 2011, 108 (32), 13118-13123. https://doi.org/10.1073/pnas.1104614108.

(23) Braun, E.; Gilmer, J.; Mayes, H. B.; Mobley, D. L.; Monroe, J. I.; Prasad, S.; Zuckerman, D. M. Best Practices for Foundations in Molecular Simulations [Article v1.0]. Living J. Comput. Mol. Sci. 2019, 1 (1), 1-28. https://doi.org/10.33011/livecoms.1.1.5957.

(24) Shan, Y.; Kim, E. T.; Eastwood, M. P.; Dror, R. O.; Seeliger, M. A.; Shaw, D. E. How Does a Drug Molecule Find Its Target Binding Site? J. Am. Chem. Soc. 2011, 133 (24), 9181-9183. https://doi.org/10.1021/ja202726y.

(25) Lindorff-Larsen, K.; Piana, S.; Dror, R. O.; Shaw, D. E. How Fast-Folding Proteins Fold. Science (80-. ). 2011, 334 (6055), 517-520. https://doi.org/10.1126/science.1208351.

(26) Freddolino, P. L.; Liu, F.; Gruebele, M.; Schulten, K. Ten-Microsecond Molecular Dynamics Simulation of a Fast-Folding WW Domain. Biophys. J. 2008, 94 (10), L75L77. https://doi.org/10.1529/biophysj.108.131565.

(27) Adcock, S. A.; McCammon, J. A. Molecular Dynamics: Survey of Methods for Simulating the Activity of Proteins. Chem. Rev. 2006, 106 (5), 1589-1615. https://doi.org/10.1021/cr040426m.

(28) Armacost, K. A.; Riniker, S.; Cournia, Z. Novel Directions in Free Energy Methods and Applications. J. Chem. Inf. Model. 2020, 60 (1), 1-5. https://doi.org/10.1021/acs.jcim.9b01174.

(29) Jorgensen, W. L.; Ravimohan, C. Monte Carlo Simulation of Differences in Free Energies of Hydration. J. Chem. Phys. 1985, 83 (6), 3050-3054. https://doi.org/10.1063/1.449208.

(30) Torrie, G. M.; Valleau, J. P. Monte Carlo Free Energy Estimates Using Non-Boltzmann Sampling: Application to the Sub-Critical Lennard-Jones Fluid. Chem. Phys. Lett. 1974, 28 (4), 578-581. https://doi.org/10.1016/0009-2614(74)80109-0.

(31) Torrie, G. M.; Valleau, J. . Nonphysical Sampling Distributions in Monte Carlo FreeEnergy Estimation: Umbrella Sampling. J. Comput. Phys. 1977, 23 (2), 187-199. https://doi.org/10.1016/0021-9991(77)90121-8.

(32) Sugita, Y.; Kitao, A.; Okamoto, Y. Multidimensional Replica-Exchange Method for FreeEnergy Calculations. J. Chem. Phys. 2000, 113 (15), 6042--6051. https://doi.org/10.1063/1.1308516.

(33) Laio, A.; Parrinello, M. Escaping Free-Energy Minima. Proc. Natl. Acad. Sci. U. S. A. 2002, 99 (20), 12562-12566. https://doi.org/10.1073/pnas.202427399.

(34) Barducci, A.; Bonomi, M.; Parrinello, M. Metadynamics. Wiley Interdiscip. Rev. Comput. Mol. Sci. 2011, 1 (5), 826-843. https://doi.org/10.1002/wcms.31.

(35) Isralewitz, B.; Gao, M.; Schulten, K. Steered Molecular Dynamics and Mechanical Functions of Proteins. Curr. Opin. Struct. Biol. 2001, 11 (2), 224-230. https://doi.org/10.1016/s0959-440x(00)00194-9. 
(36) Hamelberg, D.; Mongan, J.; McCammon, J. A. Accelerated Molecular Dynamics: A Promising and Efficient Simulation Method for Biomolecules. J. Chem. Phys. 2004, 120 (24), 11919-11929. https://doi.org/10.1063/1.1755656.

(37) Voter, A. F. Hyperdynamics: Accelerated Molecular Dynamics of Infrequent Events. Phys. Rev. Lett. 1997, 78 (20), 3908--3911. https://doi.org/10.1103/physrevlett.78.3908.

(38) Darve, E.; Rodríguez-Gómez, D.; Pohorille, A. Adaptive Biasing Force Method for Scalar and Vector Free Energy Calculations. J. Chem. Phys. 2008, 128 (14). https://doi.org/10.1063/1.2829861.

(39) Harada, R.; Kitao, A. Parallel Cascade Selection Molecular Dynamics (PaCS-MD) to Generate Conformational Transition Pathway. J. Chem. Phys. 2013, 139 (3). https://doi.org/10.1063/1.4813023.

(40) Tran, D. P.; Takemura, K.; Kuwata, K.; Kitao, A. Protein-Ligand Dissociation Simulated by Parallel Cascade Selection Molecular Dynamics. J. Chem. Theory Comput. 2018, 14 (1), 404-417. https://doi.org/10.1021/acs.jctc.7b00504.

(41) Vanden-Eijnden, E.; Venturoli, M. Markovian Milestoning with Voronoi Tessellations. $J$. Chem. Phys. 2009, 130 (19). https://doi.org/10.1063/1.3129843.

(42) Vanden-Eijnden, E.; Venturoli, M.; Ciccotti, G.; Elber, R. On the Assumptions Underlying Milestoning. J. Chem. Phys. 2008, 129 (17), 174102. https://doi.org/10.1063/1.2996509.

(43) Huber, G. A.; Kim, S. Weighted-Ensemble Brownian Dynamics Simulations for Protein Association Reactions. Biophys. J. 1996, 70 (1), 97-110. https://doi.org/10.1016/S00063495(96)79552-8.

(44) Allen, R. J.; Valeriani, C.; Rein Ten Wolde, P. Forward Flux Sampling for Rare Event Simulations. J. Phys. Condens. Matter 2009, 21 (46). https://doi.org/10.1088/09538984/21/46/463102.

(45) Tran, D. P.; Kitao, A. Dissociation Process of a MDM2/P53 Complex Investigated by Parallel Cascade Selection Molecular Dynamics and the Markov State Model. J. Phys. Chem. B 2019, 123 (11), 2469-2478. https://doi.org/10.1021/acs.jpcb.8b10309.

(46) Hata, H.; Nishihara, Y.; Nishiyama, M.; Sowa, Y.; Kawagishi, I.; Kitao, A. High Pressure Inhibits Signaling Protein Binding to the Flagellar Motor and Bacterial Chemotaxis through Enhanced Hydration. Sci. Rep. 2020, 10 (1), 3-4. https://doi.org/10.1038/s41598020-59172-3.

(47) Hata, H.; Tran, D. P.; Sobeh, M. M.; Kitao, A. Binding Free Energy of Protein/Ligand Complexes Calculated Using Dissociation Parallel Cascade Selection Molecular Dynamics and Markov State Model. Biophys. Physicobiology 2021, 18. https://doi.org/10.2142/biophysico.bppb-v18.037.

(48) Harada, R.; Shigeta, Y. Parallel Cascade Selection Molecular Dynamics Simulations for Transition Pathway Sampling of Biomolecules. In Advances in Quantum Chemistry; Elsevier Ltd, 2019; Vol. 78, pp 129-147. https://doi.org/10.1016/bs.aiq.2018.05.002.

(49) Harada, R.; Kitao, A. Nontargeted Parallel Cascade Selection Molecular Dynamics for Enhancing the Conformational Sampling of Proteins. J. Chem. Theory Comput. 2015, 11 (11), 5493-5502. https://doi.org/10.1021/acs.jctc.5b00723.

(50) Nagel, D.; Weber, A.; Stock, G. MSMPathfinder: Identification of Pathways in Markov State Models. J. Chem. Theory Comput. 2020, 16, 7882. https://doi.org/10.1021/acs.jctc.0c00774.

(51) Prinz, J. H.; Wu, H.; Sarich, M.; Keller, B.; Senne, M.; Held, M.; Chodera, J. D.; Schtte, 
C.; Noé, F. Markov Models of Molecular Kinetics: Generation and Validation. J. Chem. Phys. 2011, 134 (17). https://doi.org/10.1063/1.3565032.

(52) Noé, F. Markov Models of Molecular Kinetics. Encycl. Biophys. 2013, No. 1, 1385-1394. https://doi.org/10.1007/978-3-642-16712-6_726.

(53) Wang, Y.; Schwedes, J. F.; Parks, D.; Mann, K.; Tegtmeyer, P. Interaction of P53 with Its Consensus DNA-Binding Site. Mol. Cell. Biol. 1995, 15 (4), 2157-2165.

https://doi.org/10.1128/mcb.15.4.2157.

(54) Ho, W. C.; Fitzgerald, M. X.; Marmorstein, R. Structure of the P53 Core Domain Dimer Bound to DNA. J. Biol. Chem. 2006, 281 (29), 20494-20502. https://doi.org/10.1074/jbc.M603634200.

(55) Kitayner, M.; Rozenberg, H.; Kessler, N.; Rabinovich, D.; Shaulov, L.; Haran, T. E.; Shakked, Z. Structural Basis of DNA Recognition by P53 Tetramers. Mol. Cell 2006, 22 (6), 741-753. https://doi.org/10.1016/j.molcel.2006.05.015.

(56) Malecka, K. A.; Ho, W. C.; Ho, W. C. Crystal Structure of a P53 Core Tetramer Bound to DNA. Oncogene 2009, 28 (3), 325-333. https://doi.org/10.1038/onc.2008.400.

(57) Chen, Y.; Dey, R.; Chen, L. Crystal Structure of the P53 Core Domain Bound to a Full Consensus Site as a Self-Assembled Tetramer. Structure 2010, 18 (2), 246-256. https://doi.org/10.1016/j.str.2009.11.011.

(58) Baugh, E. H.; Ke, H.; Levine, A. J.; Bonneau, R. A.; Chan, C. S. Why Are There Hotspot Mutations in the TP53 Gene in Human Cancers? Cell Death Differ. 2018, 25 (1), 154160. https://doi.org/10.1038/cdd.2017.180.

(59) Humphrey, W.; Dalke, A.; Schulten, K. VMD: Visual Molecular Dynamics. J. Mol. Graph. 1996, 14 (1), 33-38. https://doi.org/10.1016/0263-7855(96)00018-5.

(60) Lee, J.; Hitzenberger, M.; Rieger, M.; Kern, N. R.; Zacharias, M.; Im, W. CHARMM-GUI Supports the Amber Force Fields. J. Chem. Phys. 2020, 153 (3), 035103. https://doi.org/10.1063/5.0012280.

(61) Jorgensen, W. L.; Chandrasekhar, J.; Madura, J. D.; Impey, R. W.; Klein, M. L. Comparison of Simple Potential Functions for Simulating Liquid Water. J. Chem. Phys. 1983, 79 (2), 926-935. https://doi.org/10.1063/1.445869.

(62) Maier, J. A.; Martinez, C.; Kasavajhala, K.; Wickstrom, L.; Hauser, K. E.; Simmerling, C. Ff14SB: Improving the Accuracy of Protein Side Chain and Backbone Parameters from Ff99SB. J. Chem. Theory Comput. 2015, 11 (8), 3696-3713. https://doi.org/10.1021/acs.jctc.5b00255.

(63) Zgarbová, M.; Šponer, J.; Otyepka, M.; Cheatham, T. E.; Galindo-Murillo, R.; Jurečka, P. Refinement of the Sugar-Phosphate Backbone Torsion Beta for AMBER Force Fields Improves the Description of Z- and B-DNA. J. Chem. Theory Comput. 2015, 11 (12), 5723-5736. https://doi.org/10.1021/acs.jctc.5b00716.

(64) Peters, M. B.; Yang, Y.; Wang, B.; Füsti-Molnár, L.; Weaver, M. N.; Merz, K. M. Structural Survey of Zinc-Containing Proteins and Development of the Zinc AMBER Force Field (ZAFF). J. Chem. Theory Comput. 2010, 6 (9), 2935-2947. https://doi.org/10.1021/ct1002626.

(65) Case, D. A.; Cheatham, T. E.; Darden, T.; Gohlke, H.; Luo, R.; Merz, K. M.; Onufriev, A.; Simmerling, C.; Wang, B.; Woods, R. J. The Amber Biomolecular Simulation Programs. J. Comput. Chem. 2005, 26 (16), 1668-1688. https://doi.org/10.1002/jcc.20290.

(66) Case, D. A.; Betz, R. .; Botello-Smith, W.; Cerutti, D. S.; Cheatham, T. E.; Darden, T. A.; Duke, R. E.; TGiese, T. J.; Gohlke, H.; Goetz, A. W.; Homeyer, N.; Izadi, S.; Janowski, 
P.; Kaus, J.; Kovalenko, A.; Lee, T. S. Amber 2016. Univ. California, San Fr. 2016.

Lzaguirre, J. A.; Catarello, D. P.; Wozniak, J. M.; Skeel, R. D. Langevin Stabilization of Molecular Dynamics. J. Chem. Phys. 2001, 114 (5), 2090-2098.

https://doi.org/10.1063/1.1332996.

(68) Berendsen, H. J. C.; Postma, J. P. M.; Van Gunsteren, W. F.; Dinola, A.; Haak, J. R. Molecular Dynamics with Coupling to an External Bath. J. Chem. Phys. 1984, 81 (8), 3684-3690. https://doi.org/10.1063/1.448118.

(69) Ryckaert, J. P.; Ciccotti, G.; Berendsen, H. J. C. Numerical Integration of the Cartesian Equations of Motion of a System with Constraints: Molecular Dynamics of n-Alkanes. $J$. Comput. Phys. 1977, 23 (3), 327-341. https://doi.org/10.1016/0021-9991(77)90098-5.

(70) Miyamoto, S.; Kollman, P. A. Settle: An Analytical Version of the SHAKE and RATTLE Algorithm for Rigid Water Models. J. Comput. Chem. 1992, 13 (8), 952-962. https://doi.org/10.1002/jcc.540130805.

(71) Essmann, U.; Perera, L.; Berkowitz, M. L.; Darden, T.; Lee, H.; Pedersen, L. G. A Smooth Particle Mesh Ewald Method. J. Chem. Phys. 1995, 103 (19), 8577-8593. https://doi.org/10.1063/1.470117.

(72) Adasme, M. F.; Linnemann, K. L.; Bolz, S. N.; Kaiser, F.; Salentin, S.; Haupt, V. J.; Schroeder, M. PLIP 2021: Expanding the Scope of the Protein-Ligand Interaction Profiler to DNA and RNA. Nucleic Acids Res. 2021, 1-5. https://doi.org/10.1093/nar/gkab294.

(73) Bowman, G. R.; Pande, V. S.; Noé, F. An Introduction to Markov State Models and Their Application to Long Timescale Molecular Simulation; Gregory R. Bowman and Vijay S. Pande and Frank Noe, Ed.; Springer Netherlands, 2014. https://doi.org/10.1007/978-94007-7606-7.

(74) Lloyd, S. P. Least Squares Quantization in PCM. IEEE Trans. Inf. Theory 1982, 28 (2), 129-137. https://doi.org/10.1109/TIT.1982.1056489.

(75) Arthur, D.; Vassilvitskii, S. K-Means++: The Advantages of Careful Seeding. Proc. Аnпu. ACM-SIAM Symp. Discret. Algorithms 2007, 07-09-Janu, 1027-1035.

(76) Scherer, M. K.; Trendelkamp-Schroer, B.; Paul, F.; Pérez-Hernández, G.; Hoffmann, M.; Plattner, N.; Wehmeyer, C.; Prinz, J. H.; Noé, F. PyEMMA 2: A Software Package for Estimation, Validation, and Analysis of Markov Models. J. Chem. Theory Comput. 2015, 11 (11), 5525-5542. https://doi.org/10.1021/acs.jctc.5b00743.

(77) Buch, I.; Giorgino, T.; De Fabritiis, G. Complete Reconstruction of an Enzyme-Inhibitor Binding Process by Molecular Dynamics Simulations. Proc. Natl. Acad. Sci. U. S. A. 2011, 108 (25), 10184-10189. https://doi.org/10.1073/pnas.1103547108.

(78) Doudou, S.; Sharma, R.; Henchman, R. H.; Sheppard, D. W.; Burton, N. A. Inhibitors of PIM-1 Kinase: A Computational Analysis of the Binding Free Energies of a Range of Imidazo [1,2-b] Pyridazines. J. Chem. Inf. Model. 2010, 50 (3), 368-379. https://doi.org/10.1021/ci9003514.

(79) Barber, C. B.; Dobkin, D. P.; Huhdanpaa, H. The Quickhull Algorithm for Convex Hulls. ACM Trans. Math. Softw. 1996, 22 (4), 469-483. https://doi.org/10.1145/235815.235821.

(80) Rauf, S. M. A.; Ismael, M.; Sahu, K. K.; Suzuki, A.; Koyama, M.; Tsuboi, H.; Hatakeyama, N.; Endou, A.; Takaba, H.; Del Carpio, C. A.; Kubo, M.; Miyamoto, A. The Effect of R249S Carcinogenic and H168R-R249S Suppressor Mutations on P53-DNA Interaction, a Multi Scale Computational Study. Comput. Biol. Med. 2010, 40 (5), 498508. https://doi.org/10.1016/j.compbiomed.2010.03.004.

(81) Joerger, A. C.; Hwee, C. A.; Veprintsev, D. B.; Blair, C. M.; Fersht, A. R. Structures of 
P53 Cancer Mutants and Mechanism of Rescue by Second-Site Suppressor Mutations. J. Biol. Chem. 2005, 280 (16), 16030-16037. https://doi.org/10.1074/jbc.M500179200. Joerger, A. C.; Fersht, A. R. The P53 Pathway: Origins, Inactivation in Cancer, and Emerging Therapeutic Approaches. Annu. Rev. Biochem. 2016, 85 (1), 375-404. https://doi.org/10.1146/annurev-biochem-060815-014710.

(83) Wong, K. B. O.; DeDecker, B. S.; Freund, S. M. V.; Proctor, M. R.; Bycroft, M.; Fersht, A. R. Hot-Spot Mutants of P53 Core Domain Evince Characteristic Local Structural Changes. Proc. Natl. Acad. Sci. U. S. A. 1999, 96 (15), 8438-8442. https://doi.org/10.1073/pnas.96.15.8438.

(84) Butler, J. S.; Loh, S. N. Structure, Function, and Aggregation of the Zinc-Free Form of the P53 DNA Binding Domain. Biochemistry 2003, 42 (8), 2396-2403. https://doi.org/10.1021/bi026635n.

(85) Barakat, K.; Issack, B. B.; Stepanova, M.; Tuszynski, J. Effects of Temperature on the P53-DNA Binding Interactions and Their Dynamical Behavior: Comparing the Wild Type to the R248Q Mutant. PLoS One 2011, 6 (11). https://doi.org/10.1371/journal.pone.0027651.

(86) Joerger, A. C.; Fersht, A. R. Structure-Function-Rescue: The Diverse Nature of Common P53 Cancer Mutants. Oncogene 2007, 26 (15), 2226-2242. https://doi.org/10.1038/sj.onc.1210291.

(87) Wright, J. D.; Noskov, S. Y.; Lim, C. Factors Governing Loss and Rescue of DNA Binding upon Single and Double Mutations in the P53 Core Domain. Nucleic Acids Res. 2002, 30 (7), 1563-1574. https://doi.org/10.1093/nar/30.7.1563.

(88) Suad, O.; Rozenberg, H.; Brosh, R.; Diskin-Posner, Y.; Kessler, N.; Shimon, L. J. W.; Frolow, F.; Liran, A.; Rotter, V.; Shakked, Z. Structural Basis of Restoring SequenceSpecific DNA Binding and Transactivation to Mutant P53 by Suppressor Mutations. $J$. Mol. Biol. 2009, 385 (1), 249-265. https://doi.org/10.1016/j.jmb.2008.10.063.

(89) Gomes, A. S.; Trovão, F.; Andrade Pinheiro, B.; Freire, F.; Gomes, S.; Oliveira, C.; Domingues, L.; Romão, M. J.; Saraiva, L.; Carvalho, A. L. The Crystal Structure of the R280K Mutant of Human P53 Explains the Loss of DNA Binding. Int. J. Mol. Sci. 2018, 19 (4). https://doi.org/10.3390/ijms19041184.

(90) Joerger, A. C.; Fersht, A. R. The Tumor Suppressor P53: From Structures to Drug Discovery. Cold Spring Harb. Perspect. Biol. 2010, 2 (6), 1-20.

https://doi.org/10.1101/cshperspect.a000919.

(91) Zhang, Q.; Bykov, V. J. N.; Wiman, K. G.; Zawacka-Pankau, J. APR-246 Reactivates Mutant P53 by Targeting Cysteines 124 and 277. Cell Death Dis. 2018, 9 (5). https://doi.org/10.1038/s41419-018-0463-7.

(92) He, F.; Borcherds, W.; Song, T.; Wei, X.; Das, M.; Chen, L.; Daughdrill, G. W.; Chen, J. Interaction between P53 N Terminus and Core Domain Regulates Specific and Nonspecific DNA Binding. Proc. Natl. Acad. Sci. U. S. A. 2019, 116 (18), 8859-8868. https://doi.org/10.1073/pnas.1903077116.

(93) Barros, E. P.; Demir, Ö.; Soto, J.; Cocco, M. J.; Amaro, R. E. Markov State Models and NMR Uncover an Overlooked Allosteric Loop in P53. Chem. Sci. 2021, 12 (5), 18911900. https://doi.org/10.1039/d0sc05053a.

(94) Demir, Ö.; Barros, E. P.; Offutt, T. L.; Rosenfeld, M.; Amaro, R. E. An Integrated View of P53 Dynamics, Function, and Reactivation. Curr. Opin. Struct. Biol. 2021, 67, 187194. https://doi.org/10.1016/j.sbi.2020.11.005. 
(95) Pradhan, M. R.; Siau, J. W.; Kannan, S.; Nguyen, M. N.; Ouaray, Z.; Kwoh, C. K.; Lane, D. P.; Ghadessy, F.; Verma, C. S. Simulations of Mutant P53 DNA Binding Domains Reveal a Novel Druggable Pocket. Nucleic Acids Res. 2019, 47 (4), 1637-1652. https://doi.org/10.1093/nar/gky1314. 